\title{
Age matters: The effect of onset age of video game play on task-switching abilities
}

\author{
Andree Hartanto ${ }^{1}$ - Wei Xing Toh ${ }^{1}$ - Hwajin Yang ${ }^{1}$
}

Published online: 9 February 2016

(C) The Psychonomic Society, Inc. 2016

\begin{abstract}
Although prior research suggests that playing video games can improve cognitive abilities, recent empirical studies cast doubt on such findings (Unsworth et al., 2015). To reconcile these inconsistent findings, we focused on the link between video games and task switching. Furthermore, we conceptualized video-game expertise as the onset age of active video-game play rather than the frequency of recent gameplay, as it captures both how long a person has played video games and whether the individual began playing during periods of high cognitive plasticity. We found that the age of active onset better predicted switch and mixing costs than did frequency of recent gameplay; specifically, players who commenced playing video games at an earlier age reaped greater benefits in terms of task switching than did those who started at a later age. Moreover, improving switch costs required a more extensive period of video-game experience than did mixing costs; this finding suggests that certain cognitive abilities benefit from different amounts of video game experience.
\end{abstract}

Keywords Task switching - Video game - Onset age of active video game play $\cdot$ Switch costs $\cdot$ Mixing costs $\cdot$ Executive functions

Video games are a ubiquitous entertainment medium, with an estimated $40 \%$ of adults and $83 \%$ of teenagers in the United States playing them regularly (Williams, Yee, \& Caplan, 2008). Video games often present players with challenging situations that necessitate strategic monitoring, coordination

Hwajin Yang

hjyang@smu.edu.sg

1 School of Social Sciences, Singapore Management University, 90 Stamford Road, Level 4, Singapore, Singapore 178903 of multiple task demands, and goal prioritization. For instance, first-person shooter games require players to tactically navigate a three-dimensional environment while eliminating multiple enemy targets that appear at random. Similarly, realtime strategy games typically immerse players in war-like scenarios requiring simultaneous management of several tasks, such as constructing edifices and deploying combat units to secure areas of a map or to invade and destroy opponents' territories and units. Success in such games requires continual tracking of various goals and the ability to shift flexibly from one task to another in the face of changing environmental demands. Therefore, an interesting question arises as to whether extensive video-game experience could facilitate task-switching performance (e.g., Cain, Landau, \& Shimamura, 2012; Green, Sugarman, Medford, Klobusicky, \& Bavelier, 2012).

Task switching is the ability to shift back and forth between multiple tasks or mental sets (Monsell, 2003). The efficiency of task switching can be empirically measured using taskswitching paradigms, which yield two performance indicators: switch costs and mixing costs (Rubin \& Meiran, 2005). Switch costs refer to the slower responses observed in taskswitch trials than in task-repeat trials, whereas mixing costs refer to the slower responses in task-repeat trials in mixed-task blocks than in task-repeat trials in pure blocks (Rubin \& Meiran). These two costs reflect distinct control mechanisms in task-switching performance: namely, switch costs are considered to reflect local transient control processes that govern switching from one task set to another, whereas mixing costs reflect global sustained control mechanisms responsible for monitoring and sustaining two competing task sets (Braver, Reynolds, \& Donaldson, 2003; Rubin \& Meiran, 2005). Although there is growing interest in the link between video-game experience and task switching, there are still prevailing issues warranting further examination. 
First, previous literature has largely focused on switch costs. For instance, cross-sectional studies revealed that video-game players (VGPs), as compared to non-video-game players (nVGPs), had smaller switch costs (e.g., Colzato, van Leeuwen, van den Wildenberg, \& Hommel, 2010; Green et al., 2012; Karle, Watter, \& Shedden, 2010). Consistent with these findings, longitudinal training studies have shown that playing video games for 20 to 40 hours can reduce switching costs for nVGPs (e.g., Basak, Boot, Voss, \& Kramer, 2008; Glass, Maddox, \& Love, 2013). Despite the large volume of studies showing the effects of video-game experience on switch costs, few have investigated the potential link between video-game experience and mixing costs. Consideration of this link is worthwhile because mixing costs, being closely related to global sustained control mechanisms that coordinate competing tasks (Rubin \& Meiran, 2005), would likely be involved in players' management of often highly complex video-game situations. Specifically, VGPs often must keep track of an assortment of in-game objectives requiring constant monitoring and resolution of various competing task goals. As such, it is conceivable that persistent video game play would influence mixing costs.

Another issue is the operationalization of video-game expertise. Typically, expertise has been indexed as 5 or 6 hours of gameplay per week for 6 months to a year before testing (e.g., Green et al., 2012; Latham, Patston, \& Tippett, 2013). Although some studies categorizing individuals by the frequency of recent gameplay demonstrated cognitive dissimilarities between VGPs and nVGPs (e.g., Colzato et al., 2010), others failed to do so (e.g., Irons, Remington, \& McLean, 2011; Murphy \& Spencer, 2009). Interestingly, a recent large-scale study by Unsworth et al. (2015) questioned the practice of comparing such extreme groups (e.g., VGPs vs. nVGPs) classified by the frequency of recent gameplay. Although their extreme group analyses indicated robust relationships between video-game experience and cognitive abilities on numerous cognitive control measures (e.g., Flanker or Stroop tasks), many of these reported relations were weak to nonexistent at both the task and latent-construct levels when the frequency of video-game play was employed as a continuous variable across the full range of participants. This led Unsworth et al. (2015) to conclude that prior studies with extreme group designs might have overestimated the effect sizes and found spurious relations as a result. Several factors might have accounted for these mixed results. First, the frequency of recent gameplay might not capture a sufficient history of video-game practice. In particular, this classification fails to distinguish expert adult VGPs who have been consistently playing video games since childhood, and thus would have accumulated more than 10 years of experience, from those who started 6 months ago. Second, the cognitive benefits of video-game practice may not be equivalent across the lifespan, as there may be a sensitive period for cognitive plasticity. For instance, training-induced performance gains observed in older adults appear to be less remarkable than are those in younger adults (Baltes \& Kliegl, 1992; Lövdén, Bäckman, Lindenberger, Schaefer, \& Schmiedek, 2010). Therefore, the age at which one commences video-game playing could be another contributing factor to the aforementioned mixed results. For these reasons, a more sensitive criterion is needed to accurately delineate video-game expertise.

To address these issues, our research goals are twofold. First, we examined whether the onset age of active gameplay rather than the frequency of recent gameplay is a better index of video-game expertise because the former criterion not only captures the full duration of video-game experience but also accounts for age-related discrepancies in training-dependent neurocognitive plasticity. Hence, we compared the ability of both indexes - onset age of active video-game play and frequency of recent gameplay - to predict task-switching performance. Furthermore, following Unsworth et al. (2015), we analyzed these indexes as categorical variables in an analysis of variance (ANOVA) as well as continuous variables in regression and mediation analyses. To treat the index of videogame expertise as a categorical variable in an ANOVA, the boundary between early and late onset age of active videogame play was set at 12 years old, which is widely considered to be the onset age of adolescence. This cutoff age was selected because late childhood and preadolescence - which occur prior to age 12 (Spear, 2002)-represent phases where the brain undergoes widespread development, marked by synaptic proliferation and neuronal myelination (Blakemore \& Choudhury, 2006). Furthermore, studies on the development of task-switching abilities have found that age-related changes are striking from 7 to 11 years old, with adult levels of performance being attained at around age 12 (e.g., Anderson, Anderson, Northam, Jacobs, \& Catroppa, 2001; Cepeda, Kramer, \& Gonzalez de Sather, 2001). Accordingly, the period preceding 12 years of age might signify a time when the brain is particularly sensitive to experiential inputs, such as video game experience. Using this cutoff age, we examined the effect of video-game experience - including early VGPs (EVGPs), late VGPs (LVGPs), and nVGPs-on taskswitching abilities.

Second, considering that extant video game research has explored only switch costs (e.g., Karle et al., 2010), we investigated both switch and mixing costs because they represent distinct control processes. Training-associated improvements in switch and mixing costs appear to be asymmetric; for instance, pretest-posttest reductions were larger for mixing costs than for switch costs among 8- to 10-year-old children (Karbach \& Kray, 2009). Other studies have found that mixing costs, but not switch costs, were diminished after 2 hours of training (Minear, Shah, \& Park, 2002) and eliminated after eight practice sessions (Strobach, Liepelt, Schubert, \& Kiesel, 2012). Therefore, it seems that switch 
and mixing costs are disproportionately affected by activities that require task switching, such as playing video games. Moreover, the two costs have been noted to develop differently. Although switch costs are relatively stable across age groups, mixing costs show dynamic age-related changes according to a U-shaped trajectory between 10 and 66 years old; specifically, mixing costs decrease throughout late childhood and increase approximately linearly starting from age 18 (Reimers \& Maylor, 2005). Similarly, advancing adult age (20 years and above) was found to positively correlate with mixing costs to a greater degree than with switch costs (Kray \& Lindenberger, 2000). These empirical findings point to the relatively greater malleability of mixing costs compared to switch costs in terms of training effects and age-related changes; consequently, these two costs may be dissimilarly modulated by video-game experience.

We hypothesized that the onset age of active video-game play - as reflected by the groups of EVGPs, LVGPs, and nVGPs - would predict task-switching abilities more reliably than would the frequency of recent gameplay. Specifically, EVGPs were anticipated to have both lower switch and mixing costs than nVGPs. Additionally, given that switch and mixing costs are known to be asymmetrically influenced by training, it is likely that those costs may be differently affected by video-game experience. Thus, we hypothesized that if improving switch costs requires more extensive video game experience than does improving mixing costs, EVGPs and LVGPs would show different switch costs but comparable mixing costs.

\section{Method}

\section{Participants}

One hundred and thirty-four (77 female) students from a local university in Singapore participated for extra course credit or S\$5. They were recruited through an electronic advertisement that did not reveal the study purpose. ${ }^{1}$ Our funnel questionnaire confirmed that all participants were unaware of the aim or hypotheses of the current study (Boot, Blakely, \& Simons, 2011). Based on their onset age of active video-game play, participants were divided into three groups-EVGPs $(n=43)$, LVGPs $(n=42)$, and nVGPs $(n=49)$. Participants were considered EVGPs if they had played video games actively before age 12 and LVGPs if they had played video games actively after this age. Participants who had never played any video games before were considered nVGPs. EVGPs and LVGPs who played mainly web-based puzzle

\footnotetext{
${ }^{1}$ All participants were self-reported highly proficient bilinguals; therefore, task-switching performance was not confounded by their bilingual status (Prior \& MacWhinney, 2010).
}

games (e.g., FarmVille, Tetris) were excluded because these games are highly repetitive and do not require players to constantly change strategies, rules, and goals. Both EVGPs and LVGPs played similar real-time strategy or action video games, such as Left 4 Dead, Halo, Call of Duty, Defense of the Ancients, FIFA, Grand Theft Auto, World of Warcraft, and Warcraft III. However, they differed slightly in terms of what games initiated their active gameplay. Specifically, LVGPs mainly began by playing the games that they played at the time of study, whereas EVGPs started with slightly older games such as Counter Strike, Command \& Conquer, Heroes of Might and Magic, and Super Mario. The demographic and video game playing characteristics of the participants are shown in Tables 1 and 2 (for reliability estimates, see Table 5).

\section{Materials}

Task-switching paradigm We adapted the typical taskswitching paradigm developed by Rubin and Meiran (2005) to measure the shifting function of executive control. Participants were instructed to indicate either the color (red or green) or shape (circle or triangle) of a bivalent target in response to color or shape cues. The color cue was a colored gradient and the shape cue was a row of small black shapes (size: $4.5^{\circ} \times 0.8^{\circ}$ ). The targets were a red triangle and a green circle. Participants were asked to respond as quickly and as accurately as possible using their left index finger to indicate red or a circle and their right index finger to indicate green or a triangle. The response keys were counterbalanced across participants. For each trial, a fixation cross appeared for $350 \mathrm{~ms}$, followed by a blank screen for $150 \mathrm{~ms}$. Then, the cue appeared $2.8^{\circ}$ above the fixation cross for $250 \mathrm{~ms}$. With the cue remaining on the screen, the target then appeared in the center of the screen until the participant responded. Following the response, an 850-ms intertrial interval, comprising a blank screen, was presented before the onset of the next trial. Participants received auditory feedback for incorrect responses.

Participants completed one practice block (comprising 30 trials) and six experimental blocks including two pure blocks (pure color- and shape-cue blocks, counterbalanced across participants) followed by four mixed blocks. Each experimental block comprised 50 trials. For the mixed blocks, half of the trials involved task-switching and half involved repeating the same task as in the previous trial (i.e., task-repeat trials); the presentation of such trials was randomized with a maximum of four consecutive trials of the same task.

Kaufman Brief Intelligence Test, Second Edition (KBIT-2) matrices subtest We used a computerized KBIT-2 matrices subtest (Kaufman \& Kaufman, 2004) to assess participants' nonverbal fluid intelligence. Participants were presented with a series of images depicting drawings of concrete objects or 
Table 1 Demographic, intelligence, and gaming variables

\begin{tabular}{|c|c|c|c|c|}
\hline & EVGPs $(n=43)$ & LVGPs $(n=42)$ & nVGPs $(n=49)$ & $F$ \\
\hline Age & $22.23^{\mathrm{a}}(1.94)$ & $22.19^{\mathrm{a}}(1.69)$ & $21.20^{\mathrm{b}}(1.49)$ & 5.45 \\
\hline Gender (M:F) & $29: 14^{\mathrm{a}}$ & $23: 19^{\mathrm{a}}$ & $5: 44^{\mathrm{b}}$ & $34.36^{1}$ \\
\hline Household income & $3.84^{\mathrm{a}}(2.28)$ & $3.79^{\mathrm{a}}(2.43)$ & $3.47^{\mathrm{a}}(2.29)$ & 0.34 \\
\hline Years of formal education ${ }^{2}$ & $14.32^{\mathrm{a}}(1.46)$ & $14.06^{\mathrm{a}}(2.22)$ & $14.22^{\mathrm{a}}(1.51)$ & 0.20 \\
\hline KBIT-2 (Standardized score) & $110.74^{\mathrm{a}}(11.63)$ & $113.31^{\mathrm{a}}(12.92)$ & $110.10^{\mathrm{a}}(13.17)$ & 0.79 \\
\hline Onset age of active video-game play & $8.81^{\mathrm{a}}(1.58)$ & $14.90^{\mathrm{b}}(2.35)$ & - & 198.17 \\
\hline Hours of gaming per week & $9.31^{\mathrm{a}}(9.30)$ & $8.07^{\mathrm{a}}(8.21)$ & - & 0.43 \\
\hline Self-rated video-game proficiency ${ }^{2}$ & $3.62^{\mathrm{a}}(0.82)$ & $3.33^{\mathrm{a}}(0.54)$ & - & 2.81 \\
\hline
\end{tabular}

Note. Standard deviations are shown in parentheses. Means in the same row with different superscript letters differ significantly $(p<.05)$

${ }^{1}$ Chi-square test

${ }^{2}$ Data from 18 participants were missing

abstract figures, which they then used to complete visual analogies. The standardized score had a mean of 100 and a standard deviation of 15 . The test had high internal consistency (Cronbach's $\alpha=0.91)$ and test-retest reliability $(\alpha=0.83$ ). Furthermore, the internal consistency for the KBIT-2 nonverbal standardized score for adults ranged from .87 to .93 , with a mean of .91. The KBIT-2 was also highly correlated with other fluid intelligence measures, such as the full-scale IQ of the Wechsler Adult Intelligence Scale, Third Edition $(r=.79)$.

\section{Procedure}

Participants were seated individually in an open cubicle, after which they were asked to sign an informed consent form. Then, they were asked to complete the computerized task-switching paradigm followed by the KBIT-2, followed by questionnaires assessing demographic variables and video-game exposure. We further probed participants who reported playing video games by asking about their videogame experiences, including the hours of gaming per week for the last 6 months (Green et al., 2012), the age at which

Table 2 Reaction times (RT) and accuracy rates (ACC) in the taskswitching paradigm

\begin{tabular}{lllll}
\hline & $\begin{array}{l}\text { EVGPs } \\
(n=43)\end{array}$ & $\begin{array}{l}\text { LVGPs } \\
(n=42)\end{array}$ & $\begin{array}{l}\text { nVGPs } \\
(n=49)\end{array}$ & $F$ \\
\hline ACC pure trials & $0.98^{\mathrm{a}}(0.03)$ & $0.99^{\mathrm{a}}(0.02)$ & $0.98^{\mathrm{a}}(0.02)$ & 1.14 \\
ACC repeat trials & $0.95^{\mathrm{a}}(0.11)$ & $0.96^{\mathrm{a}}(0.05)$ & $0.97^{\mathrm{a}}(0.07)$ & 0.94 \\
ACC switch trials & $0.88^{\mathrm{a}}(0.12)$ & $0.89^{\mathrm{a}}(0.08)$ & $0.89^{\mathrm{a}}(0.08)$ & 0.47 \\
RT pure trials & $399^{\mathrm{a}}(62)$ & $395^{\mathrm{a}}(71)$ & $403^{\mathrm{a}}(72)$ & 0.15 \\
RT repeat trials & $676^{\mathrm{a}}(158)$ & $696^{\mathrm{a}}(185)$ & $779^{\mathrm{b}}(182)$ & 4.51 \\
RT switch trials & $852^{\mathrm{a}}(240)$ & $937^{\mathrm{a}}(297)$ & $1049^{\mathrm{b}}(243)$ & 6.63 \\
\hline
\end{tabular}

Note. Standard deviations are shown in parentheses. Means in the same row with different superscript letters differ from each other significantly $(p<.05)$ they actively began video game play, and general video game proficiency (measured on a 5-point Likert scale). Finally, participants completed a funnel questionnaire examining their awareness of and suspicions concerning the study purpose. The entire procedure took approximately 35 to 45 minutes to complete.

\section{Results}

\section{Background measures}

The EVGPs, LVGPs, and nVGPs were matched in terms of nonverbal intelligence (as assessed by the KBIT-2), average household income (which we used as an index of socioeconomic status), and years of formal education (see Table 1), all of which have been shown to influence task-switching abilities. Thus, any group differences in task-switching abilities that we found could not be attributed to potential confounding factors.

For gaming variables, EVGPs and LVGPs differed significantly in their onset age of active video game play, $p<.001$ (see Table 1); however, they did not significantly differ in terms of hours of gameplay or self-rated video game proficiency, indicating that these groups had similar qualitative and quantitative video game experience over the past 6 months.

\section{Task-switching performance}

Response times (RT) for correct trials below $200 \mathrm{~ms}$ or above $5,000 \mathrm{~ms}$ and 2.5 standard deviations above or below each participants' mean were excluded for both pure and mixed blocks separately. Following Rubin and Meiran's (2005) procedure, we computed switch costs by subtracting the mean RT of repeat trials from that of switch 
trials in mixed blocks. We computed mixing costs by subtracting the mean RT of pure trials in pure blocks from that of repeat trials in mixed blocks. Separate analyses were then performed for switch and mixing costs. The accuracy rates were high for both pure and mixed blocks and did not differ among the three groups (see Table 2). Preliminary analyses on accuracy indicated that neither switch nor mixing costs significantly differed among EVGPs, LVGPs, and nVGPs, $F_{\mathbf{s}}<1$. Therefore, no further analyses were run on accuracy.

Mixing costs A one-way ANOVA was performed with player group (EVGPs, LVGPs, and nVGPs) as a betweenparticipants factor. There was a significant main effect of player group, $F(2,131)=5.92, p=.003, \eta_{\mathrm{p}}{ }^{2}=.083$. A subsequent planned comparison showed that EVGPs $(p=.001)$ and LVGPs $(p=.015)$ displayed significantly smaller mixing costs than did nVGPs; EVGPs and LVGPs did not significantly differ $(p=.450$; see Fig. 1$)$.

Switch costs A one-way ANOVA was performed with player group (EVGPs, LVGPs, and nVGPs) as a betweenparticipants factor. There was a significant main effect of player group, $F(2,131)=6.28, p=.002, \eta_{\mathrm{p}}{ }^{2}=.087$. A subsequent planned comparison showed that EVGPs displayed significantly smaller switch costs than did LVGPs $(p=.022)$ and nVGPs $(p=.002)$. LVGPs and nVGPs did not significantly differ, however $(p=.286)$.

\section{Regression analyses on task switching}

We performed multiple hierarchical regression analyses separately for the full sample $(N=134)$ and a composite sample of EVGPs and LVGPs $(n=85)$ to examine the unique contribution of onset age of active video-game play after controlling

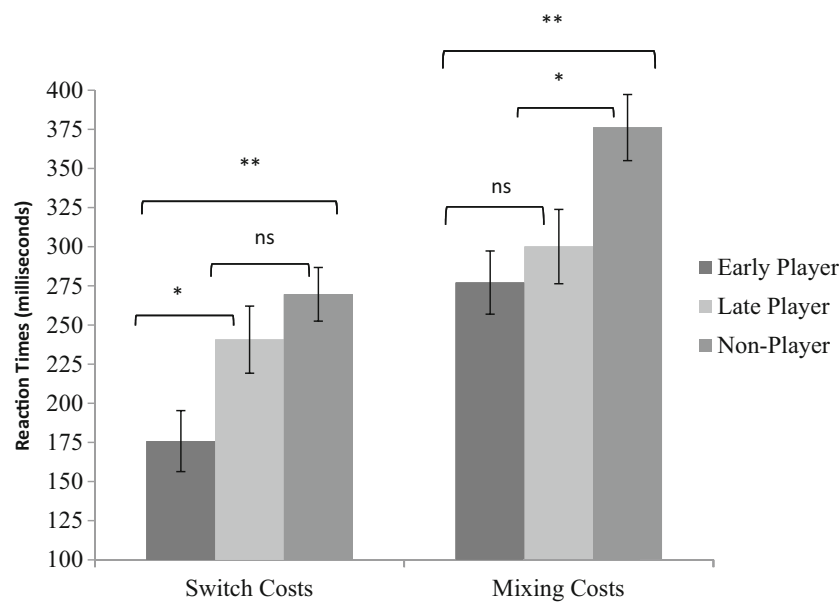

Fig. 1 Switch and mixing costs according to group of onset age of active video-game play (early, late, and nonplayers). Standard errors of the mean are presented in error bars. ${ }^{*} p<.05 .{ }^{* *} p<.001$ for gender, nonverbal intelligence, and hours of video-game playing per week (for correlation matrix, see Table 8). In the regression analyses, gender was coded dichotomously, with 0 indicating male and 1 indicating female. In all regression analyses, we entered gender, socioeconomic status, and intelligence in Step 1; hours of video-game playing per week in Step 2; and onset age of active video-game play in Step 3. The onset age of active video-game play for nVGPs was replaced with their current chronological age. ${ }^{2}$

Full-sample analysis When switch costs were regressed on the abovementioned factors, the first model explained $3.7 \%$ of the overall variance in switch costs, $F(3,130)=1.66$, $p=.180$, while the second model explained an additional $2.1 \%$ after controlling for various background measures, $\Delta F(1,129)=2.86, p=.093$. The final model explained an additional $4.2 \%$ of the variance in switch costs, $\Delta F(1,128)=$ $5.94, p=.016$. Importantly, the onset age of active video-game play emerged as the only significant predictor of switch costs, suggesting that it is a better predictor of switch costs than is hours of video-game playing (see Table 3); for correlation matrix, see Table 6 .

When the same regression analysis was performed on mixing costs, the first model explained $6.4 \%$ of the variance in mixing costs, $F(3,130)=2.98, p=.034$, whereas the second model did not explain any additional variance, $\Delta F(1,129)=$ $0.53, p=.469$. However, the final model explained an additional $3.7 \%$ of the variance in mixing costs, $\Delta F(1,128)=$ $5.32, p=.023$. Consistent with the results for switch costs, the onset age of active video-game play was the only significant predictor of mixing costs (see Table 3 ).

Composite-sample analysis For the composite sample (EVGPs and LVGPs), the hierarchical regression analysis on switch costs showed that the first model explained $1.5 \%$ of the overall variance in switch costs, $F(3,81)=$ $0.41, p=.750$, while the second model explained an additional $0.8 \%$ after controlling for background measures, $\Delta F(1,80)=0.64, p=.426$. The third model explained an additional $8.9 \%$ of the variance in switch costs, $\Delta F(1,79)=7.94, p=.006$. Consistent with the regression results of the full sample, the onset age of active videogame play emerged as the only significant predictor of switch costs, thus offering further support for its predictive ability (see Table 4); for correlation matrix, see Table 7.

In the regression analysis for mixing costs, the first model explained $3.8 \%$ of the overall variance in mixing costs, $F(3,81)=1.05, p=.374$, while the second model explained an additional $0.2 \%, \Delta F(1,80)=0.14, p=.710$.

\footnotetext{
${ }^{2}$ It is noteworthy that, despite this replacement, LVGPs $(M=14.90, S D=$ $2.35)$ and nVGPs $(M=21.20, S D=1.49)$ still significantly differed in their onset age of active video-game play, $t(89)=15.52, p<.001$.
} 
Table 3 Summary of hierarchical regression model for predicting switch and mixing costs in the full sample

\begin{tabular}{|c|c|c|c|c|c|c|c|c|}
\hline & \multicolumn{4}{|c|}{ Switch Costs } & \multicolumn{4}{|c|}{ Mixing Costs } \\
\hline & $\Delta R^{2}$ & Beta & $t$ & $p$ & $\Delta R^{2}$ & Beta & $t$ & $p$ \\
\hline Step 1 & .037 & & & & .064 & & & \\
\hline Gender & & .19 & 2.22 & .028 & & .23 & 2.66 & .009 \\
\hline Household income (SES) & & .00 & 0.03 & .973 & & -.06 & -0.65 & .516 \\
\hline Intelligence (KBIT-2) & & .02 & 0.19 & .849 & & -.08 & -0.99 & .326 \\
\hline Step 2 & .021 & & & & .004 & & & \\
\hline Gender & & .14 & 1.58 & .116 & & .21 & 2.29 & .024 \\
\hline Household income (SES) & & -.02 & -0.23 & .801 & & -.07 & -0.76 & .446 \\
\hline Intelligence (KBIT-2) & & .01 & 0.12 & .907 & & -.09 & -1.02 & .312 \\
\hline Hours of video-game playing per week & & -.15 & -1.69 & .093 & & -.07 & -0.73 & .469 \\
\hline Step 3 & .042 & & & & .037 & & & \\
\hline Gender & & .05 & 0.56 & .576 & & .12 & 1.27 & .206 \\
\hline Household income (SES) & & .00 & 0.00 & .995 & & -.50 & -0.52 & .602 \\
\hline Intelligence (KBIT-2) & & .00 & 0.04 & .971 & & -.09 & -1.11 & .269 \\
\hline Hours of video-game playing per week & & -.05 & -0.55 & .584 & & .03 & 0.29 & .771 \\
\hline Onset age of active video-game play & & .26 & 2.44 & .016 & & .24 & 2.31 & .023 \\
\hline
\end{tabular}

Note. Switch and mixing costs are in terms of RT $(\mathrm{N}=134)$

The third model explained an additional $3.9 \%$ of the variance in mixing costs, $\Delta F(1,79)=3.35, p=.071$, Notably, unlike the full-sample analysis - wherein the onset age of active video game play significantly predicted mixing costs - onset age of active video-game play moderately predicted mixing costs in this analysis $(\beta=.206, p=.071)$.

\section{Two-group analyses}

We conducted an additional analysis utilizing conventional methods of differentiating VGPs from nVGPs by the hours of video-game play. Adapted from Green et al. (2012), the criterion for being a VGP was a minimum of 5 hours per

Table 4 Summary of hierarchical regression model for predicting switch and mixing costs in the composite sample

\begin{tabular}{|c|c|c|c|c|c|c|c|c|}
\hline & \multicolumn{4}{|c|}{ Switch Costs } & \multicolumn{4}{|c|}{ Mixing Costs } \\
\hline & $\Delta R^{2}$ & Beta & $t$ & $p$ & $\Delta R^{2}$ & Beta & $t$ & $p$ \\
\hline Step 1 & .015 & & & & .038 & & & \\
\hline Gender & & .10 & 0.88 & .382 & & .10 & .91 & .365 \\
\hline Household income (SES) & & .08 & 0.72 & .473 & & .02 & .19 & .850 \\
\hline Intelligence (KBIT-2) & & .02 & 0.16 & .873 & & -.17 & -1.54 & .127 \\
\hline Step 2 & .008 & & & & .002 & & & \\
\hline Gender & & .09 & 0.80 & .429 & & .10 & 0.94 & .351 \\
\hline Household income (SES) & & .06 & 0.50 & .621 & & .03 & 0.28 & .783 \\
\hline Intelligence (KBIT-2) & & .01 & 0.10 & .924 & & -.17 & -1.50 & .138 \\
\hline Hours of video-game playing per week & & -.09 & -0.80 & .426 & & .04 & 0.37 & .710 \\
\hline Step 3 & .089 & & & & .039 & & & \\
\hline Gender & & .04 & 0.33 & .746 & & .07 & 0.62 & .539 \\
\hline Household income (SES) & & .06 & 0.51 & .613 & & .03 & 0.27 & .785 \\
\hline Intelligence (KBIT-2) & & -.05 & -0.46 & .647 & & -.21 & -1.85 & .068 \\
\hline Hours of video-game playing per week & & -.07 & -0.59 & .559 & & .06 & 0.54 & .594 \\
\hline Onset age of active video-game play & & .31 & 2.81 & .006 & & .21 & 1.83 & .071 \\
\hline
\end{tabular}

Note. Switch and mixing costs are in terms of RT $(n=85)$ 
week of video-game play over the previous 6 months. This categorization resulted in 55 VGPs and 79 nVGPs. Two independent-samples $t$ tests were conducted to compare mixing and switch costs between VGPs and nVGPs. The results revealed that VGPs $(M=288, S D=150)$ had significantly smaller mixing costs than did nVGPs $(M=344$, $S D=147), t(132)=2.16, p=.033, d=.38$. However, VGPs $(M=205, S D=148)$ had only marginally smaller switch costs than did nVGPs $(M=248, S D=120), t(132)=$ $1.88, p=.062, d=.32$.

Two separate mediation analyses were performed to clarify whether onset age of active video-game play mediated differences in mixing and switch costs between VGPs and nVGPs. In these analyses, nVGPs were coded as 0 and VGPs as 1. Multiple mediation models were estimated through the PROCESS macro (Hayes, 2009). Mediation is considered significant if the $95 \%$ bias-corrected confidence intervals for indirect effects do not encompass zero (Preacher \& Hayes, 2004). Using bias-corrected bootstrapping (with 10,000 samples), we found that onset age significantly mediated the relationship between player group and mixing costs, $95 \%$ CI $[-80.78,-14.19]$ and the relationship between player group and switch costs, $95 \%$ CI $[-86.54,-20.48]$. The residual direct effects of both models indicated full mediating effects, $p=.735$ and $p=.860$, respectively (see Fig. 2), suggesting that the differences between VGPs and nVGPs can be explained by the onset age of active video-game play.

\section{General discussion}

Our experiment yielded three major findings. First, we illuminated the relationship between onset age of active video-game play and task-switching ability, and, in doing so, we found that onset age of active video-game play serves as a better predictor of task-switching ability than does the more commonly employed index of frequency of recent video-game play. Second, unlike previous studies focusing only on switch costs, we found that VGPs had advantages in both switch and mixing costs, which adds to current understanding of task switching in the context of video games. Finally, EVGPs outperformed both LVGPs and nVGPs in terms of switch costs, whereas both EVGPs and LVGPs outperformed nVGPs in terms of mixing costs, suggesting that varying amounts of videogame experience afford dissimilar cognitive benefits to the different types of costs; in this case, mixing costs appear to be more sensitive to video-game experience than do switch costs.

Our findings highlight the superior predictive ability of onset age of active video-game play for task-switching performance: specifically, the earlier the onset of active video gaming, the greater the benefits for switch and mixing costs. Given that our participants were all of similar age, our findings related to EVGPs indicate that a longer period of video-game experience and training have greater positive impacts on cognitive plasticity. Specifically,

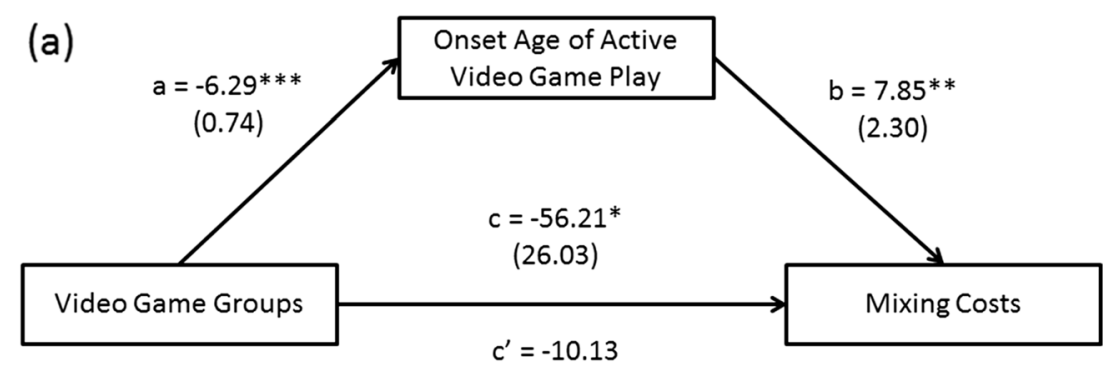

(30.98)

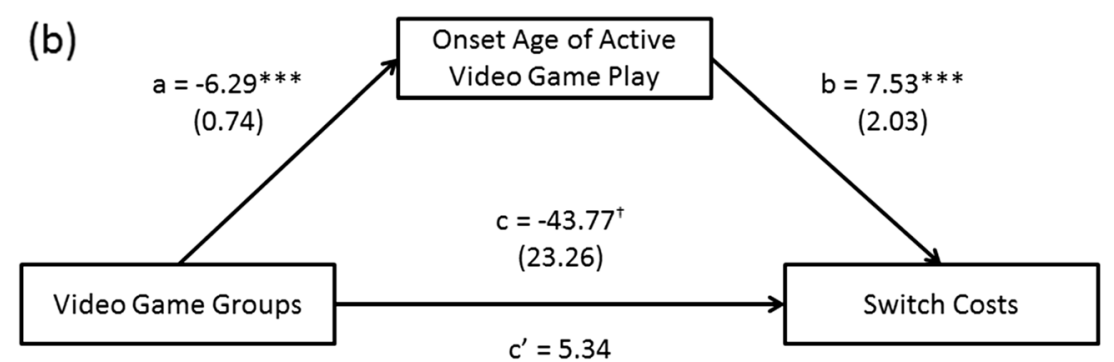

(27.37)

Fig. 2 The theoretical mediation model between player groups and either (a) mixing costs or (b) switch costs. a, b, c, and c' are the path coefficients (unstandardized regression weights with standard errors in parentheses). The $\mathrm{c}$ path coefficient represents the total effect of player group on either mixing or switch costs. The c-prime coefficient refers to the direct effect of player group on either mixing costs or switch costs. Asterisks indicate significant regression paths $\left({ }^{\dagger} p=.07, * p<.05, * * p<.01, * * * p<.05\right)$ 
EVGPs - who commenced playing video games in middle or late childhood, a period when the brain is highly plastic to environmental influences-likely experienced greater neuroanatomical changes than did other participants due to their prepubertal video-game training, which in turn lead to greater benefits in switch costs. This is consistent with electroencephalogram evidence, which has established that, compared to nVGPs, individuals who played video games before the age of 10 showed earlier N1 latencies (an event-related potential reflecting faster discrimination of visual stimuli), suggesting a more efficient operation of visual attentional resources (Latham, Patston, Westermann, Kirk, \& Tippett, 2013). Similarly, developmental studies have demonstrated that cognitive plasticity and traininginduced cognitive improvements generally decline with age (e.g., Baltes \& Kliegl, 1992). The aforementioned evidence, along with our findings, suggests that onset age of active video-game play is a crucial predictor of taskswitching ability, with earlier players enjoying more cognitive benefits compared to later players. Additionally, the finding that onset age of active video-game play is a more reliable predictor of task-switching ability than is frequency of recent gameplay accounts for, in part, the weak to nonexistent correlations in Unsworth et al.'s (2015) study, where the latter was employed as an index of video-game expertise.

The second finding of our study suggests that videogame experience amplifies task-switching ability in terms of lowered switch and mixing costs. EVGPs' advantage in switch costs is consistent with a wealth of research demonstrating a general trend in lowered switch costs for VGPs (e.g., Colzato et al., 2010; Dobrowolski, Hanusz, Sobczyk, Skorko, \& Wiatrow, 2015; Green et al., 2012), which implies superior transient control processes for the selection and activation of relevant task goals and for reconfiguring stimulus-response mappings. The reduced switch costs seen in EVGPs could be attributed to their prolonged immersion in the dynamic environment inherent to many video-game genres. For instance, while navigating from one place to another in a first-person shooter game, players might have to suspend their engagement with the current task (e.g., releasing hostages) to deal with other tasks as they arise (e.g., incoming projectile, enemy targets, or low health), requiring specific responses and strategies; furthermore, these scenarios can appear unpredictably and in rapid succession, thus allowing for limited preparatory time. Recurrent practice of abrupt attentional switching to make appropriate responses under exacting time constraints possibly relates to the reduced switch costs observed in VGPs.
Other than these well-documented advantages in switch costs, we found that VGPs had smaller mixing costs than did nVGPs, indicating that VGPs had more developed sustained-monitoring mechanisms involved in maintaining multiple task sets and resolving interference or conflicts arising from competing task sets. The complexity of video-game situations requires players to keep track of multiple, often conflicting, task demands, thereby necessitating constant awareness of task goals and resolution of competition between multiple sources of information. Furthermore, given that successful gameplay often requires sustained monitoring processes, repeated exposure to these gaming attributes potentially accounts for the lowered mixing costs seen in VGPs. Therefore, VGPs' performance likely reflects their continued video-game training, which places similar cognitive demands on them as the task-switching paradigms used in most empirical studies do.

The differing effects of onset age of active video-game play on switch and mixing costs are in line with previous studies illustrating the relative malleability of mixing costs and stability of switch costs in response to age-related changes and training effects in task-switching paradigms (e.g., Reimers \& Maylor, 2005; Strobach, Liepelt, et al., 2012). Such empirical evidence highlights the relative difficulty of reducing switch costs, particularly with short periods of training; this might account for the mixed results documented in some cross-sectional and longitudinal video game studies. For instance, Oei and Patterson (2014) found that, after 20 hours of training, reductions in switch costs were observed in only one out of four video-game training conditions. Similarly, Boot, Kramer, Simons, Fabiani, and Gratton (2008) failed to find evidence for reduced switch costs after approximately 21 hours of training. Finally, in Green et al.'s (2012) study, even with clearly longer durations (i.e., 50 hours) of training, reductions in switch costs were only marginally significant. Our findings converge with the literature suggesting that, given the durability of switch costs (Berryhill \& Hughes, 2009; Reimers \& Maylor, 2005; Strobach, Liepelt, et al., 2012), a more extensive period of training might be needed to produce notable improvements in such costs. The asymmetric outcomes of switch and mixing costs observed in our study suggest that cognitive abilities are not necessarily similarly malleable to training effects and thus may require varying amounts of training (e.g., video-game experience) for improvement.

There remain several issues requiring further clarification. The literature has shown that some video-game genres (e.g., real-time strategy, first-person shooter) afford more cognitive benefits than do others (e.g., Green et al., 2012; Strobach, Frensch, \& Schubert, 2012). Thus, it might be 
argued that the task-switching performance of the player groups (EVGPs and LVGPs) was confounded by their favored video-game genres. However, this is unlikely because we recruited participants who played either realtime strategy or action video games rather than games that are highly repetitive in nature (e.g., FarmVille). Moreover, a previous study showed that players who favored these genres did not show discrepancies in task-switching performance (Dobrowolski et al., 2015). Another question arises as to whether the onset age of active video-game play can reliably capture video-game expertise, as some players who played actively at an early age could be presently inactive. Again, this is unlikely because large-scale longitudinal studies on video-game play trends from childhood to adulthood have established that video-game players are likely to maintain or even increase their gaming frequency once they begin playing actively (e.g., Ream, Elliott, \& Dunlap, 2013). Finally, some might point to the problem of the unequal gender ratio between VGPs and nVGPs. Although it is true that women are known to be better than men at task switching (e.g., Stoet, O'Connor, Conner, \& Laws, 2013), we believe that the unbalanced gender ratio does not weaken our conclusions. Specifically, in our fullsample regression analyses, gender significantly predicted both switch and mixing costs, with men performing better than women on both types of costs. However, when the onset age of active video-game play was added to the regression model, any effect of gender on switch or mixing costs disappeared. The additional mediation analyses also indicated that the onset age of active video-game play fully mediated the relationship between gender and both switch and mixing costs. Moreover, the regression analysis on the composite sample of EVGPs and LVGPs - both of which had approximately the same gender ratio-showed, as with the full-sample analysis, that gender did not significantly predict task-switching performance. Together, this suggests that despite the potential importance of gender in task switching, its effect may be negligible in consideration of other video-game-related factors (e.g., onset age of active video-game play).

Our study is not without its limitations, particularly those regarding causality. Additionally, given that we employed only a single measure of task switching, it would be worthwhile to examine the robustness of our findings using variants of the task-switching paradigm, such as the number-letter switching task. It would also be beneficial to examine whether the positive effect of onset age of active video-game play extends to other aspects of executive functioning (e.g., inhibitory control, working memory). Nevertheless, we addressed several prominent methodological and statistical issues not previously considered in video-game research. First, we dealt with potential preexisting group differences by controlling for demographic factors such as fluid intelligence, educational background, and socioeconomic status. Second, VGPs and nVGPs had comparable accuracy in the task-switching paradigm, differing only in RT. This suggests that there was no speed-accuracy trade-off (McDermott, Bavelier, \& Green, 2014). Third, by reanalyzing our data using continuous independent variables (i.e., frequency of recent videogame play, onset age of active video-game play) across all participants in the regression and mediation analyses, we circumvented problems related to extreme group designs, such as overestimated effect sizes and increased likelihood of Type I errors (Unsworth et al., 2015).

In summary, the findings of our study revealed that variations in task-switching performance exist even among VGPs; specifically, earlier onset age of active video-game play confers greater cognitive benefits. Furthermore, videogame experience appears to have distinct effects on taskswitching ability in terms of mixing and switch costs, with the former being more "trainable" than the latter. Future research should consider how video-game experience interacts with innate factors (e.g., genetic predispositions, personality traits) and other forms of training (e.g., musical training, team sports) that might make individuals differentially receptive to video-game practice. For example, it has been shown that genetic variations in the regulation of neurotransmitters in the prefrontal cortex modulate the effects of video-game training on task-switching performance: After 10 hours of video-game training, switch costs were reduced among individuals carrying the valine allele but not among those with the methionine allele (Colzato, van den Wildenberg, \& Hommel, 2014). A deeper understanding of how various factors might combine with video-game training to promote task-switching performance holds practical utility for populations interested in improving their cognitive flexibility. In particular, video-game training could be viably incorporated into intervention regimens targeting age- and disease-related cognitive decline and impairments. Indeed, some studies have already showed promising results (e.g., Basak et al., 2008; Belchior et al., 2013).

Acknowledgments This study was supported by a grant awarded to Hwajin Yang by Singapore Management University through a research grant (14-C242-SMU-031) from the Ministry of Education Academy Research Fund Tier 1. A preliminary version of this research was presented at the 27th American Psychological Science Annual Convention. We thank Choi Hae Song, Gaurav Singh, Johannes Judy, Matthew Tham, and $\mathrm{Lu}$ Yizhen for their assistance in data collection and coding. 


\section{Appendix A}

Table 5 Descriptive statistics and reliability estimates for task-switching paradigm

\begin{tabular}{|c|c|c|c|c|c|}
\hline & $M$ & $S D$ & Skewness & Kurtosis & Reliability $^{1}$ \\
\hline \multicolumn{6}{|l|}{ Task-switching reaction times } \\
\hline Switch costs ${ }^{2}$ & 230 & 134 & 0.68 & 0.68 & .84 \\
\hline Mixing costs ${ }^{3}$ & 321 & 150 & 0.45 & -0.38 & .95 \\
\hline Pure trials & 399 & 68 & 1.26 & 2.99 & .99 \\
\hline Repeat trials & 720 & 180 & 0.56 & 0.30 & .97 \\
\hline Switch trials & 950 & 271 & 0.51 & 0.42 & .98 \\
\hline \multicolumn{6}{|l|}{ Task-switching accuracy } \\
\hline Switch costs ${ }^{2}$ & -.07 & .06 & -0.79 & 0.41 & .63 \\
\hline Mixing costs ${ }^{3}$ & -.02 & .07 & -4.74 & 28.33 & .90 \\
\hline Pure trials & .98 & .02 & -2.05 & 6.56 & .60 \\
\hline Repeat trials & .96 & .07 & -4.66 & 25.55 & .94 \\
\hline Switch trials & .89 & .10 & -1.98 & 5.05 & .91 \\
\hline \multicolumn{6}{|l|}{ Video game experience (full sample) } \\
\hline Onset age of active video game play & 15.25 & 5.46 & -0.06 & -1.36 & - \\
\hline Hours of gameplay per week (last 6 months) & 5.52 & 8.13 & 2.33 & 6.45 & - \\
\hline \multicolumn{6}{|l|}{ Video game experience (composite sample) } \\
\hline Onset age of active video game play ${ }^{4}$ & 11.82 & 3.65 & 0.36 & -0.49 & - \\
\hline Hours of gameplay per week (last 6 months) & 8.70 & 8.76 & 1.96 & 4.44 & - \\
\hline Self-rated video game proficiency & 3.48 & 0.70 & 0.35 & -0.12 & - \\
\hline
\end{tabular}

${ }^{1}$ Reliability was estimated using the split-half procedure based on even- and odd-numbered trials that were corrected using the Spearman-Brown prophecy formula

${ }^{2}$ Switch costs were calculated by subtracting the mean RT (or accuracy) of repeat trials from that of switch trials in mixed blocks

${ }^{3}$ Mixing costs were calculated by subtracting the mean RT (or accuracy) of pure trials in pure blocks from that of repeat trials in mixed blocks

${ }^{4}$ For the full sample, onset age of active video game play for nVGPs was replaced with their current chronological age

\section{Appendix B}

Table 6 Zero-order correlations among video game and demographic variables in the full sample

\begin{tabular}{|c|c|c|c|c|c|c|c|c|}
\hline & & 1 & 2 & 3 & 4 & 5 & 6 & 7 \\
\hline 1. & Onset age of active video game play & - & & & & & & \\
\hline 2. & Hours of gameplay per Week (last 6 months) & $-.484^{*}$ & - & & & & & \\
\hline 3. & Age & -.160 & $.191^{*}$ & - & & & & \\
\hline 4. & Gender & $.473^{*}$ & $-.300^{*}$ & $-.542^{*}$ & - & & & \\
\hline 5. & Monthly household income & -.069 & -.133 & -.137 & -.091 & - & & \\
\hline 6. & KBIT-2 & .034 & -.032 & -.096 & -.021 & -.015 & - & \\
\hline 7. & Years of formal education ${ }^{1}$ & .048 & -.055 & $.609^{*}$ & -.021 & -.110 & -.138 & - \\
\hline
\end{tabular}

Note. The onset age of active video game play for nVGPs was replaced with their current chronological age. Self-rated video game proficiency for the full sample was not available because video game proficiency data were not obtained from nVGPs

${ }^{1}$ Multiple imputation techniques were applied to 18 missing values by using Rubin's rule, which combines parameter estimates across the 5 imputed data sets obtained by multiple imputation procedures (Rubin, 1987). ${ }^{*} p<.05$ 
Table 7 Zero-order correlations among video game and demographic variables in the composite sample

\begin{tabular}{|c|c|c|c|c|c|c|c|c|c|}
\hline & & 1 & 2 & 3 & 4 & 5 & 6 & 7 & 8 \\
\hline 1. & Onset age of active video game play & - & & & & & & & \\
\hline 2. & Hours of Gameplay per week (last 6 months) & -.119 & - & & & & & & \\
\hline 3 & Self-rated video game proficiency & $-.231^{*}$ & $.258^{*}$ & - & & & & & \\
\hline 4. & Age & -.030 & .068 & .095 & - & & & & \\
\hline 5. & Gender & .179 & -.064 & $-.287^{*}$ & $-.564^{*}$ & - & & & \\
\hline 6. & Monthly household income & .025 & $-.248^{*}$ & .006 & -.145 & -.122 & - & & \\
\hline 7. & KBIT-2 & .206 & -.106 & .058 & $-.234^{*}$ & .007 & .110 & - & \\
\hline 8. & Years of formal education & -.036 & -.069 & -.131 & $.630^{*}$ & -.081 & -.133 & -.226 & \\
\hline
\end{tabular}

Note. The composite sample includes EVGPs and LVGPs $(n=85)$

$* p<.05$

Table 8 Zero-order correlations between video game experience and task switching

\begin{tabular}{|c|c|c|c|c|}
\hline & \multicolumn{2}{|c|}{ Switch Costs } & \multicolumn{2}{|c|}{ Mixing Costs } \\
\hline & RT & $\mathrm{ACC}$ & RT & $\mathrm{ACC}$ \\
\hline \multicolumn{5}{|l|}{ Full Sample $(n=134)$} \\
\hline Onset age of active video game play & $.307^{*}$ & .020 & $.285^{*}$ & .110 \\
\hline Hours of gameplay per week (last 6 months) & $-.194^{*}$ & .013 & -.116 & -.122 \\
\hline \multicolumn{5}{|l|}{ Composite Sample $(n=85)$} \\
\hline Onset age of active video game play & $.317^{*}$ & .013 & .170 & .056 \\
\hline Hours of gaming per week (last 6 months) & -.113 & -.048 & .045 & -.083 \\
\hline Self-rated video game proficiency & -.120 & .121 & -.057 & -.041 \\
\hline
\end{tabular}

Note. The composite sample includes EVGPs and LVGPS. For the full sample, the onset age of active gameplay for nVGPs was replaced with their current chronological age. Self-rated video game proficiency for the full sample was not available because video game proficiency data were not obtained from nVGPs. ${ }^{*} p<.05$

\section{References}

Anderson, V. A., Anderson, P., Northam, E., Jacobs, R., \& Catroppa, C. (2001). Development of executive functions through late childhood and adolescence in an Australian sample. Developmental Neuropsychology, 20(1), 385-406. doi:10.1207/ S15326942DN2001 5

Baltes, P. B., \& Kliegl, R. (1992). Further testing of limits of cognitive plasticity: Negative age differences in a mnemonic skill are robust. Developmental Psychology, 28(1), 121-125. doi:10.1037/00121649.28.1.121

Basak, C., Boot, W. R., Voss, M. W., \& Kramer, A. F. (2008). Can training in a real-time strategy video game attenuate cognitive decline in older adults? Psychology and Aging, 23(4), 765-777. doi: 10.1037/a0013494

Belchior, P., Marsiske, M., Sisco, S. M., Yam, A., Bavelier, D., Ball, K., \& Mann, W. C. (2013). Video game training to improve selective visual attention in older adults. Computers in Human Behavior, 29(4), 1318-1324. doi:10.1016/j.chb.2013.01.034
Berryhill, M. E., \& Hughes, H. C. (2009). On the minimization of task switch costs following long-term training. Attention, Perception, \& Psychophysics, 71(3), 503-514. doi:10.3758/ APP.71.3.503

Blakemore, S. J., \& Choudhury, S. (2006). Development of the adolescent brain: Implications for executive function and social cognition. Journal of Child Psychology and Psychiatry, 47(3), 296-312. doi: 10.1111/j.1469-7610.2006.01611.x

Boot, W. R., Blakely, D. P., \& Simons, D. J. (2011). Do action video games improve perception and cognition? Frontiers in Psychology, 2, 226. doi:10.3389/fpsyg.2011.00226

Boot, W. R., Kramer, A. F., Simons, D. J., Fabiani, M., \& Gratton, G. (2008). The effects of video game playing on attention, memory, and executive control. Acta Psychologica, 129(3), 387-398. doi:10. 1016/j.actpsy.2008.09.005

Braver, T. S., Reynolds, J. R., \& Donaldson, D. I. (2003). Neural mechanisms of transient and sustained cognitive control during task switching. Neuron, 39(4), 713-726. doi:10.1016/S0896-6273(03) 00466-5 
Cain, M. S., Landau, A. N., \& Shimamura, A. P. (2012). Action video game experience reduces the cost of switching tasks. Attention, Perception, \& Psychophysics, 74(4), 641-647. doi:10.3758/ s13414-012-0284-1

Cepeda, N. J., Kramer, A. F., \& Gonzalez de Sather, J. (2001). Changes in executive control across the life span: Examination of taskswitching performance. Developmental Psychology, 37(5), 715. doi:10.1037/0012-1649.37.5.715

Colzato, L. S., van den Wildenberg, W. P. M., \& Hommel, B. (2014). Cognitive control and the COMT Val158Met polymorphism: Genetic modulation of videogame training and transfer to taskswitching efficiency. Psychological Research, 78(5), 670-678. doi: 10.1007/s00426-013-0514-8

Colzato, L. S., van Leeuwen, P. J., van den Wildenberg, W., \& Hommel, B. (2010). DOOM'd to switch: Superior cognitive flexibility in players of first person shooter games. Frontiers in Psychology, 1, 8. doi:10.3389/fpsyg.2010.00008

Dobrowolski, P., Hanusz, K., Sobczyk, B., Skorko, M., \& Wiatrow, A. (2015). Cognitive enhancement in video game players: The role of video game genre. Computers in Human Behavior, 44, 59-63. doi: 10.1016/j.chb.2014.11.051

Glass, B. D., Maddox, W. T., \& Love, B. C. (2013). Real-time strategy game training: Emergence of a cognitive flexibility trait. PloS One, 8(8), e70350. doi:10.1371/journal.pone.0070350

Green, C. S., Sugarman, M. A., Medford, K., Klobusicky, E., \& Bavelier, D. (2012). The effect of action video game experience on taskswitching. Computers in Human Behavior, 28(3), 984-994. doi: 10.1016/j.chb.2011.12.020

Hayes, A. F. (2009). Beyond Baron and Kenny: Statistical mediation analysis in the new millennium. Communication Monographs, 76(4), 408-420. doi:10.1080/03637750903310360

Irons, J. L., Remington, R. W., \& McLean, J. P. (2011). Not so fast: Rethinking the effects of action video games on attentional capacity. Australian Journal of Psychology, 63(4), 224-231. doi:10.1111/j. 1742-9536.2011.00001.x

Kaufman, A. S., \& Kaufman, N. L. (2004). Kaufman brief intelligence test (2nd ed.). Circle Pines: American Guidance Service.

Karbach, J., \& Kray, J. (2009). How useful is executive control training? Age differences in near and far transfer of task-switching training. Developmental Science, 12(6), 978-990. doi:10.1111/j.1467-7687. 2009.00846.x

Karle, J. W., Watter, S., \& Shedden, J. M. (2010). Task switching in video game players: Benefits of selective attention but not resistance to proactive interference. Acta Psychologica, 134(1), 70-78. doi:10. 1016/j.actpsy.2009.12.007

Kray, J., \& Lindenberger, U. (2000). Adult age differences in task switching. Psychology and Aging, 15(1), 126. doi:10.1037/08827974.15.1.126

Latham, A. J., Patston, L. L. M., \& Tippett, L. J. (2013). Just how expert are "expert" video-game players? Assessing the experience and expertise of video-game players across "action" video-game genres. Frontiers in Psychology, 4, 941. doi:10.3389/fpsyg.2013.00941

Latham, A. J., Patston, L. L., Westermann, C., Kirk, I. J., \& Tippett, L. J. (2013). Earlier visual N1 latencies in expert video-game players: a temporal basis of enhanced visuospatial performance? PloS One, $8(9), \mathrm{e} 75231$

Lövdén, M., Bäckman, L., Lindenberger, U., Schaefer, S., \& Schmiedek, F. (2010). A theoretical framework for the study of adult cognitive plasticity. Psychological Bulletin, 136(4), 659-676. doi:10.1037/ a0020080

McDermott, A. F., Bavelier, D., \& Green, C. S. (2014). Memory abilities in action video game players. Computers in Human Behavior, 34, 69-78. doi:10.1016/j.chb.2014.01.018

Minear, M., Shah, P., \& Park, D. (2002). Training task-shifting skills in older adults. Poster presented at the 2002 meeting of the Cognitive Aging Conference, Atlanta, GA.

Monsell, S. (2003). Task switching. Trends in Cognitive Sciences, 7(3), 134-140.

Murphy, K., \& Spencer, A. (2009). Playing video games does not make for better visual attention skills. Journal of Articles in Support of the Null Hypothesis, 6(1), 1-20. Retrieved from http://www.jasnh.com/ pdf/Vol6-No1.pdf

Oei, A. C., \& Patterson, M. D. (2014). Playing a puzzle video game with changing requirements improves executive functions. Computers in Human Behavior, 37, 216-228. doi:10.1016/j.chb.2014.04.046

Preacher, K. J., \& Hayes, A. F. (2004). SPSS and SAS procedures for estimating indirect effects in simple mediation models. Behavior Research Methods, Instruments, \& Computers, 36(4), 717-731. doi:10.3758/bf03206553

Prior, A., \& MacWhinney, B. (2010). A bilingual advantage in task switching. Bilingualism: Language and Cognition, 13(2), 253262. doi:10.1017/s1366728909990526

Ream, G. L., Elliott, L. C., \& Dunlap, E. (2013). Trends in video game play through childhood, adolescence, and emerging adulthood. Psychiatry Journal, 1-7. doi:10.1155/2013/301460

Reimers, S., \& Maylor, E. A. (2005). Task switching across the life span: Effects of age on general and specific switch costs. Developmental Psychology, 41(4), 661-671. doi:10.1037/0012-1649.41.4.661

Rubin, D. B. (1987). Multiple imputation for nonresponse in surveys. New York: Wiley. doi:10.1002/9780470316696

Rubin, O., \& Meiran, N. (2005). On the origins of the task mixing cost in the cuing task-switching paradigm. Journal of Experimental Psychology. Learning, Memory, and Cognition, 31(6), 1477-1491. doi:10.1037/0278-7393.31.6.1477

Spear, L. P. (2002). Alcohol's effects on adolescents. Alcohol Research and Health, 26(4), 287-291.

Stoet, G., O’Connor, D. B., Conner, M., \& Laws, K. R. (2013). Are women better than men at multi-tasking? BMC Psychology, 1(1), 18. doi: $10.1186 / 2050-7283-1-18$

Strobach, T., Frensch, P. A., \& Schubert, T. (2012a). Video game practice optimizes executive control skills in dual-task and task switching situations. Acta Psychologica, 140(1), 13-24. doi:10.1016/j.actpsy. 2012.02.001

Strobach, T., Liepelt, R., Schubert, T., \& Kiesel, A. (2012b). Task switching: Effects of practice on switch and mixing costs. Psychological Research, 76(1), 74-83. doi:10.1007/s00426-0110323-x

Unsworth, N., Redick, T. S., McMillan, B. D., Hambrick, D. Z., Kane, M. J., \& Engle, R. W. (2015). Is playing video games related to cognitive abilities? Psychological Science, 26(6), 759-774. doi:10.1177/ 0956797615570367

Williams, D., Yee, N., \& Caplan, S. E. (2008). Who plays, how much, and why? Debunking the stereotypical gamer profile. Journal of Computer-Mediated Communication, 13(4), 993-1018. doi:10. 1111/j.1083-6101.2008.00428.x 